\title{
Walrasian Price Equilibrium
}

\author{
Anna Nagurney \\ Isenberg School of Management \\ University of Massachusetts \\ Amherst, MA 01003
}

(C) 2002 
In this lecture the focus is on general economic equilibrium problems, in particular, Walrasian price or pure exchange equilibria. This problem has been extensively studied in the economics literature dating to Walras (1874); see also Wald (1951), Debreu (1959), and MasColell (1985). Specifically, in this chapter we apply the powerful theory of variational inequalities to both the qualitative analysis of general economic equilibria as well as to their computation.

\section{Network Equilibrium Equivalence}

We first briefly review the pure exchange economic equilibrium model and give its variational inequality formulation. Some fundamental theoretical results are then presented. The network equilibrium formulation is also given here.

Consider a pure exchange economy with $l$ commodities, and with column price vector $p$ taking values in $R_{+}^{l}$ and with components $p_{1}, \ldots, p_{l}$. Denote the induced aggregate excess demand function $z(p)$, which is a row vector with components $z_{1}(p), \ldots, z_{l}(p)$. Assume that $z(p)$ is generally defined on a subcone $C$ of $R_{+}^{l}$ which contains the interior $R_{++}^{l}$ of $R_{+}^{l}$, that is, $R_{++} \subset C \subset R_{+}^{l}$. 
Hence, the possibility that the aggregate excess demand function may become unbounded when the price of a certain commodity vanishes is allowed. As usual, assume that $z(p)$ satisfies Walras' law, that is, $\langle z(p), p\rangle=0$ on $C$ and that $z(p)$ will be homogeneous of degree zero in $p$ on $C$, that is, $z(\alpha p)=z(p)$ for all $p \in C, \alpha>0$. 
Because of homogeneity, one may normalize the prices so that they take values in the simplex:

$$
S^{l}=\left\{p: p \in R_{+}^{l}, \sum_{i=1}^{l} p_{i}=1\right\},
$$

and, therefore, one may restrict the aggregate excess demand function to the intersection $D$ on $S^{l}$ with $C$. Let

$$
S_{+}^{l}=\left\{p: p>0, p \in S^{l}\right\},
$$

and note that $S_{+}^{l} \subset D \subset S^{l}$.

As is standard in general economic equilibrium theory, assume that

(i) The function $z(p): D \mapsto R^{l}$ is continuous.

(ii) The function $z(p)$ satisfies Walras' law

$$
\langle z(p), p\rangle=0, \quad \forall p \in D .
$$


The definition of a Walrasian equilibrium is now stated.

\section{Definition 1 (Walrasian Price Equilibrium)}

A price vector $p^{*} \in R_{+}^{l}$ is a Walrasian equilibrium price vector if

$$
z\left(p^{*}\right) \leq 0
$$

The following theorem establishes that Walrasian price vectors can be characterized as solutions of a variational inequality.

\section{Theorem 1 (Variational Inequality Formulation of Walrasian Equilibrium)}

A price vector $p^{*} \in D$ is a Walrasian equilibrium if and only if it satisfies the variational inequality

$$
\left\langle z\left(p^{*}\right), p-p^{*}\right\rangle \leq 0, \quad \forall p \in S^{l} .
$$


Proof: Observe, first, that variational inequality (5) is equivalent to

$$
\left\langle z\left(p^{*}\right), p\right\rangle \leq 0, \quad \forall S^{l},
$$

by virtue of Walras' law (3). Assume now that $p^{*} \in D$ is a Walrasian equilibrium price vector, that is, it satisfies (4). Then, clearly, (6) holds true. On the other hand, assuming that (6) holds for all $p \in S^{l}$ and selecting $p=$ $(0,0, \ldots, 1,0, \ldots, 0)$ with a 1 at the $i$-th position, one concludes that $z_{i}\left(p^{*}\right) \leq 0 ; i=1, \ldots, l$.

The proof is complete.

Recall the geometric interpretation of a variational inequality. The interpretation in the above variational inequality model is that $z\left(p^{*}\right)$ is "orthogonal" to the set $S^{l}$ and points away from the set $S^{l}$. In particular, the result is the following.

\section{Proposition 1}

A price vector $p^{*}$ is a Walrasian equilibrium, or, equivalently, a solution of the above variational inequality if and only if it is a fixed point of the projection map

$$
G(p)=P_{S^{l}}(p+\rho z(p)),
$$

Where $\rho>0$ and $P_{S^{\prime}}$ indicates the projection map onto the compact convex set $S^{l}$. 
Note that if the aggregate excess demand function $z(p)$ is defined and is continuous on all of $S^{l}$, that is, $D=S^{l}$, then the existence of at least one Walrasian equilibrium price vector in $S^{l}$ follows immediately.

However, since $D$ is not necessarily compact, this result no longer holds. Nevertheless, one may still apply this theorem to deduce that $z(p)$ exhibits the needed behavior near the boundary of $S^{l}$, in particular, that at least some of the components of $z(p)$ become in a sense "large" as $p$ approaches points on the boundary of $S^{l}$ that are not contained in $D$. Several existence proofs of this type can be found in Border (1985). We now provide the result proven in Dafermos (1990).

\section{Theorem 2 (Existence)}

Assume that the aggregate excess demand function $z(p)$ satisfies the following assumption: If $S^{l} \backslash D$ is nonempty, then with any sequence $\left\{p_{n}\right\}$ in $S_{+}^{l}$ which converges to a point of $S^{l} \backslash D$ there is associated a point $\bar{p} \in S_{+}^{l}$, generally dependent on $\left\{p_{n}\right\}$, such that the sequence $\left\langle z\left(p_{n}\right), \bar{p}\right\rangle$ contains infinitely many positive terms. Then there exists a Walrasian equilibrium price vector $p^{*} \in D$. 
A special class of aggregate excess demand functions is now considered, for which the following result holds true.

\section{Theorem 3}

Assume that $-z(p)$ is continuous and monotone on $D$. Then $p^{*} \in D$ is a Walrasian equilibrium price vector if and only if

$$
\left\langle z(p), p-p^{*}\right\rangle \leq 0, \quad \forall p \in D,
$$

or, equivalently, if and only if

$$
\left\langle z(p), p^{*}\right\rangle \geq 0, \quad \forall p \in D .
$$

An immediate consequence of the above is the following.

\section{Corollary 1}

Assume that $-z(p)$ is continuous and monotone on $D$ and $D$ is compact. Then the set of Walrasian equilibrium price vectors is a convex subset of $D$. 
The uniqueness issue is now investigated; in particular, if one strengthens the monotonicity assumption somewhat, one obtains the following result.

\section{Theorem 4 (Uniqueness Under Strict Monotonic- ity)}

Assume that $-z(p)$ is strictly monotone on $D$, that is,

$$
\left\langle z\left(p^{1}\right)-z\left(p^{2}\right), p^{1}-p^{2}\right\rangle<0, \quad \forall p^{1}, p^{2} \in D, p^{1} \neq p^{2} .
$$

Then there exists at most a single Walrasian price equilibrium vector $p^{*}$.

Proof: Assume that $p^{*} \in D$ and $q^{*} \in D$ are Walrasian price equilibrium vectors. Then each vector satisfies, respectively, variational inequality (5), that is,

$$
\left\langle z\left(p^{*}\right), p-p^{*}\right\rangle \leq 0, \quad \forall p \in S^{l}
$$

and

$$
\left\langle z\left(q^{*}\right), p-q^{*}\right\rangle \leq 0, \quad \forall p \in S^{l} .
$$

Letting $p=q^{*}$ in (10), and $p=p^{*}$ in (11), and adding the two resulting inequalities, one obtains

$$
\left\langle z\left(p^{*}\right)-z\left(q^{*}\right), p^{*}-q^{*}\right\rangle \geq 0 .
$$

But, by the definition of strict monotonicity on $D$, cannot hold unless $p^{*}=q^{*}$. The proof is complete. 


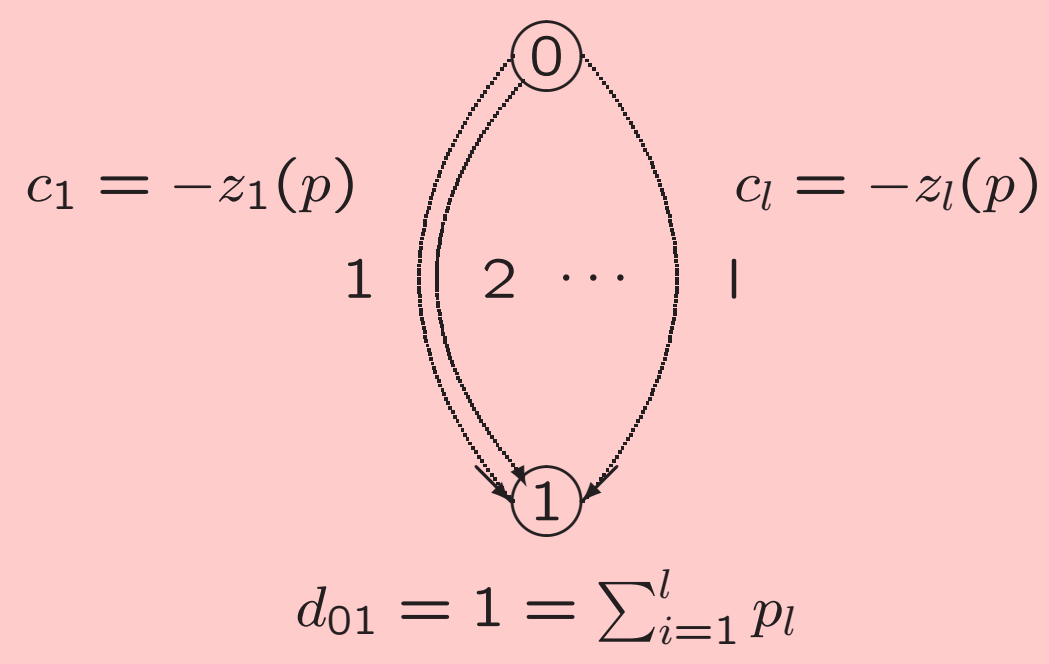

Network formulation of the pure exchange economy 
Below it is established that the variational inequality model (5) for the Walrasian price equilibrium problem is isomorphic to a network equilibrium model with special structure.

Consider the following network equilibrium problem: A network is given consisting of a single origin node $0, a$ single destination node 1 , and with a single origin/destination pair $(0,1)$. There are $l$ links connecting the origin/destination pair $(0,1)$ (cf. Figure 1 ). A fixed O/D demand $d_{01}$ is assumed given. Let $f_{i}$ denote the flow passing through link $i ; i=1, \ldots, l$, and let $c_{i}$ be the user cost associated with link $i ; i=1, \ldots, l$.

Group the link loads into a column vector $f \in R_{l}$, and the costs into a row vector $c \in R_{l}$. Assume the general situation that a cost on a link may depend upon the entire link load pattern, that is, $c_{i}=c_{i}(f)$.

Then $f^{*}$ is a user equilibrium pattern if and only if no user has any incentive to change his/her path, which in the model corresponds to a link. 
In other words, mathematically, there exists an ordering of the links $n_{i} ; i=1, \ldots, l$, such that

$$
c_{n_{1}}\left(f^{*}\right), \ldots, c_{n_{s}}\left(f^{*}\right)=\lambda \leq c_{n_{s+1}}\left(f^{*}\right) \leq \ldots \leq c_{n_{l}}\left(f^{*}\right)
$$

where

$$
f_{n_{i}}^{*}\left\{\begin{array}{l}
>0, \quad i=1, \ldots, s \\
=0, \quad i=s+1, \ldots, l .
\end{array}\right.
$$

(13) is equivalent to the following statement:

A vector $f^{*} \in K$ is a user equilibrium load pattern if and only if it is a solution to the variational inequality

$$
\left\langle c\left(f^{*}\right), f-f^{*}\right\rangle \geq 0, \quad \forall f \in K,
$$

where

$$
K \equiv\left\{f: f \geq 0, \sum_{i=1}^{l} f_{i}=d_{01}\right\}
$$


The relationship between the variational inequality and the above network equilibrium problem is now established. Consider the demand

$$
d_{01}=1
$$

the link flow pattern

$$
f \equiv p
$$

and the user cost

$$
c(\cdot) \equiv-z(\cdot)
$$

The equilibrium condition of the network with the cost vector defined in (15) is:

$$
z_{i}\left(p^{*}\right)\left\{\begin{array}{lll}
=\lambda, & \text { if } & p_{i}^{*}>0 \\
\leq \lambda, & \text { if } & p_{i}^{*}=0 .
\end{array}\right.
$$

Multiplying now the above inequalities by $p_{i}^{*} ; i=1, \ldots, l$, and summing then the resulting equalities, and using Walras' law, one obtains

$$
\lambda=\left\langle z\left(p^{*}\right), p^{*}\right\rangle=0 .
$$

Hence, the equilibrium condition (16) of the above network with the cost function defined in (15) is identical to the equilibrium condition (4) of the pure exchange economy, with Walras' law (3) holding.

Furthermore, variational inequality (14) which governs the network equilibrium problem described above coincides with variational inequality (5) which governs the Walrasian price equilibrium problem. 
Recall now the costless migration equilibrium model developed in the migration lecture. In the case of a single class of migrant, the resulting model's network equilibrium representation is identical to the network equilibrium representation of the pure exchange economy problem depicted in Figure 1. Hence, these two models are isomorphic.

However, in the migration model, the flows on the network links correspond to populations at the respective locations, whereas in the pure exchange model, the flows on the links correspond to prices.

The costs on the migration network correspond to the disutility functions, whereas the costs on the Walrasian network correspond to excess supply functions. 
We will subsequently show how the special network structure underlying the Walrasian price equilibrium model can be exploited for computational purposes. The results in light of the discussion above, are applicable to the migration equilibrium model as well. 


\section{Sensitivity Analysis}

ow the sensitivity analysis properties of Walrasian price equilibria are investigated. In particular, the sensitivity of the solution price vector to changes in the data is examined. The variational inequality approach allows one to perform sensitivity analysis even when the equilibrium lies on the boundary.

First, consider the comparison of two equilibria. We begin with the statement of the following lemma, which will be useful in the further analysis.

\section{Lemma 1}

Let $z$ and $z^{*}$ denote two aggregate excess demand functions, and let $p$ and $p^{*}$ denote, respectively, their associated Walrasian equilibrium price vectors. Then

$$
\left\langle z^{*}\left(p^{*}\right)-z(p), p^{*}-p\right\rangle \geq 0 .
$$

Moreover, when $-z$ is strictly monotone (without any monotonicity assumption imposed on $z^{*}$ ), then

$$
\left\langle z^{*}\left(p^{*}\right)-z\left(p^{*}\right), p^{*}-p\right\rangle \geq 0,
$$

with equality holding only when $p=p^{*}$. 
Proof: Since $p$ and $p^{*}$ are both Walrasian price equilibrium vectors, by Theorem 1 , they must satisfy, respectively, the variational inequalities

$$
\begin{gathered}
\langle z(p), q-p\rangle \leq 0, \quad \forall q \in S^{l}, \\
\left\langle z^{*}\left(p^{*}\right), q-p^{*}\right\rangle \leq 0, \quad \forall q \in S^{l} .
\end{gathered}
$$

Letting $q=p^{*}$ in (19), and $q=p$ in (20), and summing the two resulting inequalities, one obtains (17).

From (17), one has that

$$
\left\langle z^{*}\left(p^{*}\right)-z(p)+z\left(p^{*}\right)-z\left(p^{*}\right), p^{*}-p\right\rangle \geq 0 .
$$

When $-z(p)$ is strictly monotone, (21) yields

$$
\left\langle z^{*}\left(p^{*}\right)-z\left(p^{*}\right), p^{*}-p\right\rangle \geq-\left\langle z\left(p^{*}\right)-z(p), p^{*}-p\right\rangle \geq 0 ;
$$

and, therefore, (18) follows with equality holding only when $p=p^{*}$. 
Applying Walras' law to (18) and (19) above, one concludes with the following.

\section{Corollary 2}

Let $z$ and $z^{*}$ denote two aggregate excess demand functions, and let $p$ and $p^{*}$ be their corresponding Walrasian price vectors. Then

$$
\left\langle z^{*}\left(p^{*}\right), p\right\rangle+\left\langle z(p), p^{*}\right\rangle \leq 0
$$

and, assuming that $-z$ is strictly monotone

$$
\left\langle z^{*}\left(p^{*}\right), p\right\rangle \leq\left\langle z\left(p^{*}\right), p\right\rangle,
$$

with equality holding only when $p=p^{*}$.

Now another sensitivity analysis result is stated.

\section{Theorem 5}

Let $z$ and $z^{*}$ denote two aggregate excess demand functions, and $p$ and $p^{*}$ the corresponding Walrasian price equilibrium vectors. Assume that $z$ satisfies the strong monotonicity assumption

$$
\left\langle z\left(p^{1}\right)-z\left(p^{2}\right), p^{1}-p^{2}\right\rangle \leq-\alpha\left\|p^{1}-p^{2}\right\|^{2}, \quad \forall p^{1}, p^{2} \in D,
$$

where $\alpha>0$. Then

$$
\left\|p^{*}-p\right\| \leq \frac{1}{\alpha}\left\|z^{*}\left(p^{*}\right)-z\left(p^{*}\right)\right\| .
$$


Proof: From Lemma 1 one has that (17) holds, and from (17) that

$$
\left\langle z^{*}\left(p^{*}\right)-z(p)+z\left(p^{*}\right)-z\left(p^{*}\right), p^{*}-p\right\rangle \geq 0 .
$$

But from the strong monotonicity condition (25), (27) yields

$$
\left\langle z^{*}\left(p^{*}\right)-z\left(p^{*}\right), p^{*}-p\right\rangle \geq-\left\langle z\left(p^{*}\right)-z(p), p^{*}-p\right\rangle \geq \alpha\left\|p^{*}-p\right\|^{2} .
$$

By virtue of the Schwartz inequality, (28) yields

$$
\alpha\left\|p^{*}-p\right\|^{2} \leq\left\|z^{*}\left(p^{*}\right)-z\left(p^{*}\right)\right\|\left\|p^{*}-p\right\|,
$$

from, whence, (26) follows and the proof is complete. 


\section{A General Walrasian Iterative Scheme}

Now a general iterative scheme for the computation of Walrasian price equilibria is described. The scheme is based on the general iterative scheme of Dafermos and, at each iteration, the scheme allows for the exploitation of the special network structure depicted in Figure 1. In the study of algorithms and their convergence, the standard assumption in the economics literature (cf. Scarf (1973)) is that the aggregate excess demand function $z(p)$ is well-defined and continuous on all of $S^{l}$. Here this assumption is also made. The scheme is as follows.

\section{The Walrasian Iterative Scheme}

Construct a smooth function $g(p, q): S^{l} \times S^{l} \mapsto R^{l}$ with the following properties:

(i) $g(p, p)=-z(p), \quad \forall p \in S^{l}$,

(ii) for every $p, q \in S^{l}$, the $l \times l$ matrix $\nabla_{p} g(p, q)$ is positive definite.

Any smooth function $g(p, q)$ with the above properties generates the following algorithm.

\section{Step 0: Initialization}

Start with some $p^{0} \in S^{l}$. Set $k:=1$. 
Step 1: Construction and Computation

Compute $p^{k}$ by solving the variational inequality

$$
\left\langle g\left(p^{k}, p^{k-1}\right)^{T}, p-p^{k}\right\rangle \geq 0, \quad \forall p \in S^{l} .
$$

\section{Step 2: Convergence Verification}

If $\left|p^{k}-p^{k-1}\right| \leq \epsilon$, with $\epsilon>0$, a prespecified tolerance, then stop; otherwise, set $k:=k+1$, and go to Step 1 . 
For simplicity, and easy reference, denote the above variational inequality by $\mathrm{VI}^{k}\left(g, S^{l}\right)$. Since $\nabla_{p} g(p, q)$ is positive definite, $\mathrm{VI}^{k}\left(g, S^{l}\right)$ admits a unique solution $p^{k}$. Thus, we obtain a well-defined sequence $\left\{p^{k}\right\}$. It is easy to verify that if the sequence $\left\{p^{k}\right\}$ is convergent, say $p^{k} \rightarrow p^{*}$, as $k \rightarrow \infty$, then $p^{*}$ is an equilibrium price vector, that is, it is a solution of variational inequality (9.5). In fact, on account of the continuity of $g(p, q), \mathrm{VI}^{k}\left(g, S^{l}\right)$ yields

$$
\begin{aligned}
& -\left\langle z\left(p^{*}\right), p-p^{*}\right\rangle=\left\langle g\left(p^{*}, p^{*}\right)^{T}, p-p^{*}\right\rangle \\
= & \lim _{k \rightarrow \infty}\left\langle g\left(p^{k}, p^{k-1}\right)^{T}, p-p^{k}\right\rangle \geq 0, \forall p \in S^{l}
\end{aligned}
$$

so that $p^{*}$ is a solution of the original variational inequality (5). 
We now establish conditions on $g(p, q)$ which guarantee that the sequence $\left\{p^{k}\right\}$ is convergent. For simplicity, let $|\cdot|$ denote the usual Euclidean norm in the space $R^{l}$ and let $\|\cdot\|$ denote the norm of the operator $Q: G^{\frac{1}{2}} V \mapsto R^{l}$,

$$
\|Q\|=\sup _{u \in G^{\frac{1}{2}} V,|u|=1}|Q u|
$$

where

$$
G(p, q)=\frac{1}{2}\left(\nabla_{p} g(p, q)+\nabla_{p} g^{T}(p, q)\right),
$$

which is positive definite.

$$
V=\left\{v: v \in R^{l}, \sum_{i=1}^{l} v_{i}=0\right\}
$$

and

$$
G^{\frac{1}{2}} V=\left\{u: u=G^{\frac{1}{2}}(p, q) v, v \in V\right\} .
$$

The conditions for convergence are now presented.

\section{Theorem 7 (Convergence)}

Assume that

$$
\left\|G^{-\frac{1}{2}}\left(p^{1}, q^{1}\right) \nabla_{q} g\left(p^{2}, q^{2}\right) G^{-\frac{1}{2}}\left(p^{3}, q^{3}\right)\right\|<1,
$$

for all $\left(p^{1}, q^{1}\right),\left(p^{2}, q^{2}\right),\left(p^{3}, q^{3}\right) \in S^{l}$. Then the sequence $\left\{p^{k}\right\}$ obtained by solving $\mathrm{VI}^{k}\left(g, S^{l}\right)$ is Cauchy on $S^{l}$. 
Proof: Let $p=p^{k+1}$ for $\operatorname{VI}^{k}\left(g, S^{l}\right)$, that is,

$$
\left\langle g\left(p^{k}, p^{k-1}\right)^{T}, p^{k+1}-p^{k}\right\rangle \geq 0,
$$

and let $p=p^{k}$ for $\mathrm{VI}^{k+1}\left(g, S^{l}\right)$, that is,

$$
\left\langle g\left(p^{k+1}, p^{k}\right)^{T}, p^{k}-p^{k+1}\right\rangle \geq 0 .
$$

Adding (38) and (39), one obtains

$$
\left\langle\left(g\left(p^{k}, p^{k-1}\right)-g\left(p^{k+1}, p^{k}\right)\right)^{T}, p^{k+1}-p^{k}\right\rangle \geq 0,
$$

or

$$
\begin{aligned}
& \left\langle\left(g\left(p^{k+1}, p^{k}\right)-g\left(p^{k}, p^{k}\right)\right)^{T}, p^{k+1}-p^{k}\right\rangle \\
\leq & \left\langle\left(g\left(p^{k}, p^{k-1}\right)-g\left(p^{k}, p^{k}\right)\right)^{T}, p^{k+1}-p^{k}\right\rangle .
\end{aligned}
$$

By the Mean Value Theorem, there exists a $t \in(0,1)$, such that

$$
\begin{gathered}
\left\langle\left(g\left(p^{k+1}, p^{k}\right)-g\left(p^{k}, p^{k}\right)\right)^{T}, p^{k+1}-p^{k}\right\rangle=\left(p^{k+1}-p^{k}\right)^{T} \\
\nabla_{p} g\left(t p^{k}+(1-t) p^{k+1}, p^{k}\right)\left(p^{k+1}-p^{k}\right)
\end{gathered}
$$

or

$$
\begin{gathered}
\left\langle\left(g\left(p^{k+1}, p^{k}\right)-g\left(p^{k}, p^{k}\right)\right)^{T}, p^{k+1}-p^{k}\right\rangle \\
=\frac{1}{2}\left(p^{k+1}-p^{k}\right)^{T} \cdot\left(\nabla_{p} g\left(t p^{k}+(1-t) p^{k+1}, p^{k}\right)+\right. \\
\nabla_{p}^{T} g\left(t p^{k}+(1-t) p^{k+1}, p^{k}\right) \cdot\left(p^{k+1}-p^{k}\right) .
\end{gathered}
$$


Let $G_{k}$ be defined as

$G_{k}=\frac{1}{2}\left(\nabla_{p} g\left(t p^{k}+(1-t) p^{k+1}, p^{k}\right)+\nabla_{p}^{T} g\left(t p^{k}+(1-t) p^{k+1}, p^{k}\right)\right)$.

Observe that $G_{k}$ is symmetric and positive definite. Using now (41), (43), and (44) yields

$$
\begin{array}{r}
\left\langle\left(p^{k+1}-p^{k}\right)^{T}, G_{k}\left(p^{k+1}-p^{k}\right\rangle\right. \\
\leq\left\langle g\left(p^{k}, p^{k-1}\right)-g\left(p^{k}, p^{k}\right), p^{k+1}-p^{k}\right\rangle .
\end{array}
$$

Define now the inner product on $V$ as

$$
\left(v_{1}, v_{2}\right)_{k}=v_{1}^{T} G_{k} v_{2}, \quad \forall v_{1}, v_{2} \in V
$$

which induces the norm

$$
|v|_{k}=\left(v^{T} G_{k} v\right)^{\frac{1}{2}}=\left|G_{k}^{\frac{1}{2}} v\right|, \quad \forall v \in V .
$$

By applying the Mean Value Theorem, (45) yields

$$
\begin{gathered}
\left|p^{k+1}-p^{k}\right|_{k}^{2} \leq\left(p^{k-1}-p^{k}\right)^{T} G_{k-1}^{\frac{1}{2}} G_{k-1}^{-\frac{1}{2}} \\
\nabla_{q} g\left(p^{k}, s p^{k}+(1-s) p^{k-1}\right) G_{k}^{-\frac{1}{2}} G_{k}^{\frac{1}{2}}\left(p^{k+1}-p^{k}\right)
\end{gathered}
$$

for $s \in(0,1)$. 
Using now the Schwarz inequality and condition (37) yields

$$
\begin{gathered}
\left|p^{k+1}-p^{k}\right|_{k}^{2} \leq\left|G_{k-1}^{\frac{1}{2}}\left(p^{k}-p^{k-1}\right)\right| \times \| G_{k-1}^{-\frac{1}{2}} \\
\nabla_{q} g\left(p^{k}, s p^{k}+(1-s) p^{k-1}\right) G_{k}^{-\frac{1}{2}} \| \times\left|G_{k}^{\frac{1}{2}}\left(p^{k+1}-p^{k}\right)\right| \\
=\left|p^{k}-p^{k-1}\right|_{k-1}\left\|G_{k-1}^{-\frac{1}{2}} \nabla_{q} g\left(p^{k}, s p^{k}+(1-s) p^{k-1}\right) G_{k}^{-\frac{1}{2}}\right\| \\
\times\left|p^{k+1}-p^{k}\right|_{k} .
\end{gathered}
$$

Hence,

$$
\left|p^{k+1}-p^{k}\right|_{k} \leq \gamma\left|p^{k}-p^{k-1}\right|_{k-1}, \quad k=1,2, \ldots,
$$

where $\gamma$ is the maximum over the compact set $S^{l}$ of the lefthand side of (37).

From (50) one obtains

$$
\left|p^{k+1}-p^{k}\right|_{k} \leq \gamma\left|p^{k}-p^{k-1}\right|_{k-1} \leq \ldots \leq \gamma^{k}\left|p^{1}-p^{0}\right|_{0} .
$$


On the other hand, since $G_{k} ; k=1,2, \ldots$, is nonsingular, for every $(p, q) \in S^{l} \times S^{l}$, there is a $\beta>0$ such that

$$
\begin{gathered}
\left|p^{k+1}-p^{k}\right| \leq \beta^{-1}\left|p^{k+1}-p^{k}\right|_{k}, \\
\forall p^{k+1}, p^{k} ; k=0,1,2, \ldots
\end{gathered}
$$

Therefore, (51) yields

$$
\begin{aligned}
& \left|p^{k+r}-p^{k}\right| \leq \sum_{i=k}^{k+r-1}\left|p^{i+1}-p^{i}\right| \leq \beta^{-1} \sum_{i=k}^{k+r-1}\left|p^{i+1}-p^{i}\right|_{i} \\
& \leq \beta^{-1}\left|p^{1}-p^{0}\right|_{0} \sum_{i=k}^{k+r-1} \gamma^{i}=\beta^{-1}\left|p^{1}-p^{0}\right|_{0} \frac{\gamma^{k}}{1-\gamma}
\end{aligned}
$$

which shows that $\left\{p^{k}\right\}$ is a Cauchy sequence in $S^{l}$ and the proof is complete.

Of course, the resulting variational inequality subproblems, in this case, $\mathrm{VI}^{k}\left(g, S^{l}\right)$, should be constructed in such a way so that each is easy to solve. We emphasize this point later in discussing the projection method and the relaxation method, and the specific equilibration algorithms for the solution of the embedded subproblems. 


\section{Proposition 2}

Assume that the Jacobian matrix $\nabla_{p} g(p, q)$ is also symmetric. Then a necessary condition for (35) to hold is that the Jacobian matrix $\nabla z(p)$ is negative definite over $V$ for any $p \in S^{l}$, that is,

$$
v^{T} \nabla z(p) v<0, \quad \forall v \in V, v \neq 0, \forall p \in S^{l} .
$$

The above condition implies that the function $-z(p)$ is strictly monotone on $S^{l}$, that is,

$$
\left\langle z\left(p^{1}\right)-z\left(p^{2}\right), p^{1}-p^{2}\right\rangle<0, \quad \forall p^{1}, p^{2} \in S^{l}, p^{1} \neq p^{2} .
$$

Proof: Assume that condition (37) holds and select

$$
p^{1}=p^{2}=p^{3}=q^{1}=q^{2}=q^{3} \text {. }
$$

Note that

$$
-\nabla_{p} z(p)=\nabla_{p} g(p, p)+\nabla_{q} g(p, p) .
$$

Therefore, (37) takes the form

$$
\left\|I+G^{-\frac{1}{2}}(p, p) \nabla_{p} z(p) G^{-\frac{1}{2}}(p, p)\right\|<1 .
$$

Set

$$
B(p)=G^{-\frac{1}{2}}(p, p) \nabla_{p} z(p) G^{-\frac{1}{2}}(p, p) .
$$


Substituting now (57) into (56) and expanding the lefthand side of (56), we obtain

$$
\begin{aligned}
& \|I+B\|^{2}=\sup _{u \in G^{\frac{1}{2}} V,|u|=1}|(I+B) u|^{2} \\
& =\sup _{u \in G^{\frac{1}{2}} V,|u|=1} u^{T}(I+B)^{T}(I+B) u \\
& =\sup _{u}\left(1+2 u^{T} B u+u^{T} B^{T} B u\right)<1
\end{aligned}
$$

or,

$$
2 u^{T} B u<-u^{T} B^{T} B u .
$$

Since $u=G^{\frac{1}{2}}(p, p) v$, (59) yields

$$
\begin{aligned}
& 2 v^{T} \nabla_{p} z(p) v<-v^{T} \nabla_{p}^{T} z(p) G^{-\frac{1}{2}}(p, p) G^{-\frac{1}{2}}(p, p) \nabla_{p} z(p) v \\
& =-\left|G^{-\frac{1}{2}}(p, p) \nabla_{p} z(p) v\right|^{2} \leq 0, \quad \forall v \in V, p \in S^{l}, v \neq 0 .
\end{aligned}
$$

Hence, $\nabla_{p} z(p)$ is negative definite over $V$ for any $p \in S^{l}$. The proof is complete. 
Note that since $z(p)$ is homogeneous of degree zero $\nabla z(p)$ cannot be positive definite. Therefore, $z(p)$ is never strictly monotone on a set containing a segment of the ray originating from the origin of the $l$-dimensional space. However, it can be strictly monotone on the $l-1$ dimensional simplex $S^{l}$ (see, e. g., Dafermos (1990)). 


\section{The Projection Method}

It is now demonstrated that the general iterative scheme induces a projection method and, subsequently, that it also induces the relaxation method. In the context of the pure exchange model both the projection method and the relaxation method resolve the variational inequality problem into simpler subproblems, which can then be solved using equilibration algorithms.

We refer to the equilibration algorithms, respectively, as WPEA, to denote "Walrasian Projection Equilibration Algorithm," and WREA, to denote "Walrasian Relaxation Equilibration Algorithm," and state each of these, for completeness.

Note that the network subproblems induced by the projection method are characterized by linear link cost functions, whereas those induced by the relaxation method are, in general, nonlinear. 
The projection method corresponds to the choice

$$
g(p, q)=-z(q)+\frac{1}{\rho} G(p-q),
$$

where $\rho$ is a positive scalar and $G$ is a fixed, symmetric positive definite matrix. In this case properties (i) and (ii) are satisfied. In fact,

(i). $g(p, q)=-z(p)+\frac{1}{\rho} G(p-p)=-z(p)$,

(ii). $\nabla_{p} g(p, q)=\rho^{-1} G$, is positive definite and symmetric.

Condition (37) then takes the form

$$
\left\|I+\rho G^{-\frac{1}{2}} \nabla_{p} z(p) G^{-\frac{1}{2}}\right\|<1 .
$$

The following lemma give conditions under which (61) is satisfied.

\section{Lemma 2}

If $-z(p)$ is strongly monotone on $S^{l}$, then condition (61) is satisfied. 
With such a selected $g(p, q)$, each subproblem $\mathrm{VI}^{k}\left(g, S^{l}\right)$ is isomorphic to the network equilibrium problem with linear link cost functions. In particular, one may select $G$ to be the diagonal positive definite matrix of the form

$$
\left[\begin{array}{ccc}
\alpha_{1} & \cdots & 0 \\
\vdots & \ddots & \vdots \\
0 & \cdots & \alpha_{l}
\end{array}\right]
$$

where $\alpha_{i} ; i=1,2, \ldots, l$, is any positive number. A natural choice is to have $\alpha_{i}=-\left.\frac{\partial z_{i}}{\partial p_{i}}\right|_{p^{0}} ; i=1,2, \ldots, l$, in which case $\mathrm{VI}^{k}\left(g, S^{l}\right)$ is then isomorphic to the separable network equilibrium problem depicted in Figure 2. Such a problem can be solved in closed form using an equilibration algorithm. Here, for completeness, its resolution in the context of the Walrasian price model is presented. First, some notation is given. 


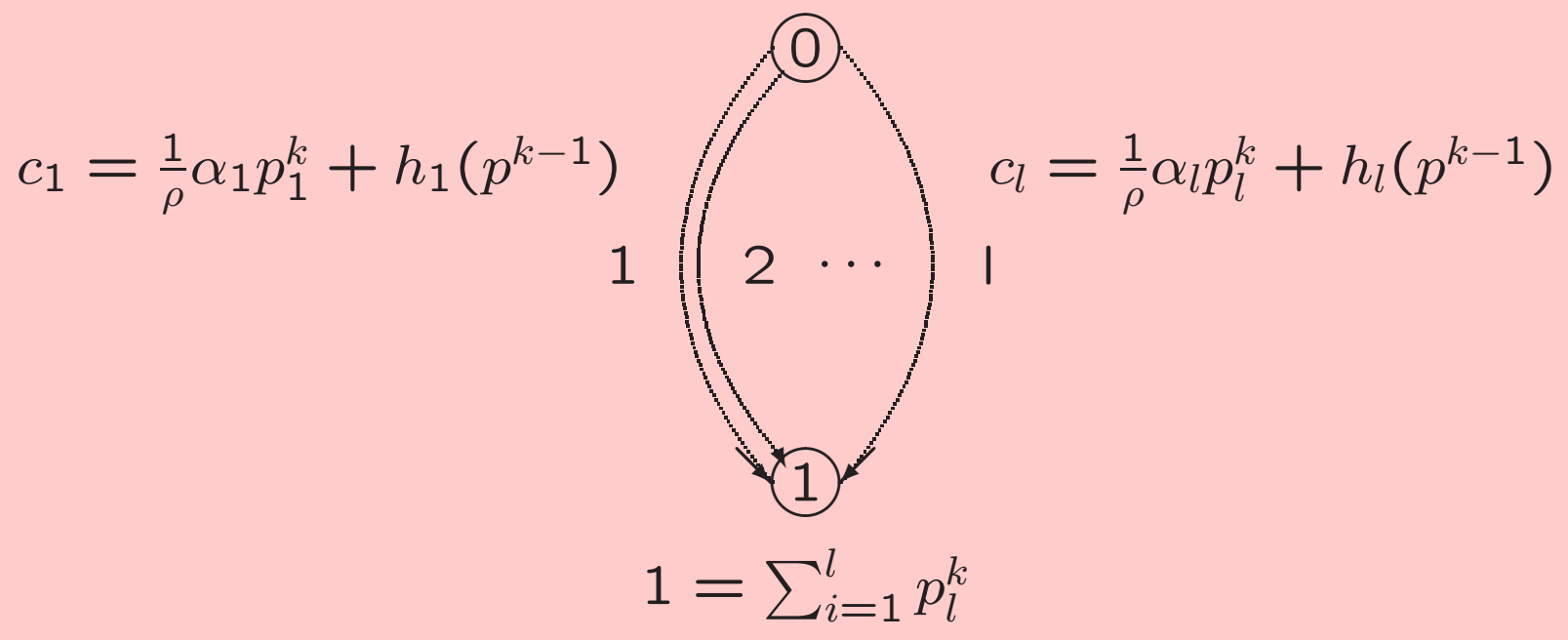

Network equilibrium representation of subproblem induced by the projection method 
Let the components of $g\left(p, p^{k-1}\right)$ be given by $g_{i}\left(p, p^{k-1}\right)=-z_{i}\left(p^{k-1}\right)+\frac{1}{\rho} \alpha_{i}\left(p_{i}-p_{i}^{k-1}\right), \quad i=1,2, \ldots, l$, and define

$$
h_{i}\left(p^{k-1}\right)=-z_{i}\left(p^{k-1}\right)-\frac{1}{\rho} \alpha_{i} p_{i}^{k-1}, \quad i=1,2, \ldots, l .
$$

Then

$$
g_{i}\left(p, p^{k-1}\right)=\frac{1}{\rho} \alpha_{i} p_{i}+h_{i}\left(p^{k-1}\right), \quad i=1,2, \ldots, l .
$$

The Walrasian Projection Equilibration Algorithm is stated immediately following.

\section{WPEA}

\section{Step 0: Sort}

Sort the numbers $h_{i} ; i=1,2, \ldots, l$, in nondescending order, and relabel them accordingly. Assume, henceforth, that they are relabeled. Also, define $h_{l+1} \equiv \infty$. Set $L:=1$. 


\section{Step 1: Computation}

Compute

$$
\lambda^{L}=\frac{1+\rho \sum_{i=1}^{L} \frac{h_{i}}{\alpha_{i}}}{\rho \sum_{i=1}^{L} \frac{1}{\alpha_{i}}} .
$$

\section{Step 2: Evaluation}

If $h_{L}<\lambda^{L} \leq h_{L+1}$, let $s=L, \lambda=\lambda^{L}$, and go to Step 3; otherwise, set $L:=L+1$, and go to Step 1 .

\section{Step 3: Update}

Set

$$
\begin{aligned}
p_{i}^{k} & =\frac{\rho}{\alpha_{i}}\left(\lambda-h_{i}\right), \quad i=1,2, \ldots, s \\
p_{i}^{k} & =0, \quad i=s+1, s+2, \ldots, l .
\end{aligned}
$$

The algorithm converges in a finite number of steps. 


\section{The Relaxation Method}

The relaxation method corresponds to the choice $g_{i}(p, q)=-z_{i}\left(q_{1}, \ldots, q_{i-1}, p_{i}, q_{i+1}, \ldots, q_{l}\right), \quad \forall i=1,2, \ldots, l$. In this case properties (i) and (ii) are also satisfied. In fact,

(i). $g(p, p)=-z(p)$,

(ii). $\nabla_{p} g(p, q)=\left[\begin{array}{ccc}-\frac{\partial z_{1}}{\partial p_{1}} & \cdots & 0 \\ \vdots & \ddots & \vdots \\ 0 & \cdots & -\frac{\partial z_{l}}{\partial p_{l}}\end{array}\right]$ is a diagonal matrix.

By recalling the properties of the aggregate excess demand function $z(p)$, one deduces that it is reasonable to assume that

$$
\frac{\partial z_{i}}{\partial p_{i}}<0, \quad \forall i=1,2, \ldots, l .
$$

Hence, $\nabla_{p} g(p, q)$ is positive definite. Furthermore,

$$
\nabla_{q} g(p, q)=\left[\begin{array}{cccc}
0 & \frac{\partial z_{1}}{\partial p_{2}} & \cdots & \frac{\partial z_{1}}{\partial p_{l}} \\
\frac{\partial z_{2}}{\partial p_{1}} & 0 & \cdots & \frac{\partial z_{2}}{\partial p_{l}} \\
\vdots & \vdots & \ddots & \vdots \\
\frac{\partial z_{l}}{\partial p_{1}} & \cdots & \frac{\partial z_{l}}{\partial p_{l-1}} & 0
\end{array}\right]
$$


and

$$
\begin{gathered}
\nabla_{p} g^{-\frac{1}{2}}\left(p_{1}, q_{1}\right) \nabla_{q} g\left(p_{2}, q_{2}\right) \nabla_{p} g^{-\frac{1}{2}}\left(p_{3}, q_{3}\right) \\
=\left[\begin{array}{ccc}
0 & \frac{\partial z_{1}}{\partial p_{2}}\left(-\frac{\partial z_{1}}{\partial p_{1}}\right)^{-\frac{1}{2}}\left(-\frac{\partial z_{2}}{\partial p_{2}}\right)^{-\frac{1}{2}} & \ldots \\
\frac{\partial z_{2}}{\partial p_{1}}\left(-\frac{\partial z_{2}}{\partial p_{2}}\right)^{-\frac{1}{2}}\left(-\frac{\partial z_{2}}{\partial p_{2}}\right)^{-\frac{1}{2}} & 0 & \cdots \\
\vdots & \vdots & \ddots \\
\frac{\partial z_{l}}{\partial p_{1}}\left(-\frac{\partial z_{1}}{\partial p_{1}}\right)^{-\frac{1}{2}}\left(-\frac{\partial z_{l}}{\partial p_{l}}\right)^{-\frac{1}{2}} & \cdots & \cdots
\end{array}\right] .
\end{gathered}
$$


We now state the following.

\section{Theorem 8}

Let

$$
-\frac{\partial z_{T}}{\partial p_{T}}=\min _{i}\left\{-\frac{\partial z_{i}}{\partial p_{i}}\right\}
$$

and assume that

$$
-\frac{\partial z_{T}}{\partial p_{T}}>\sum_{k \neq i} \frac{\partial z_{i}}{\partial p_{k}}\left(-\frac{\partial z_{k}}{\partial p_{k}}\right)^{-\frac{1}{2}}\left(-\frac{\partial z_{T}}{\partial p_{T}}\right)^{\frac{1}{2}}, \quad \forall i=1,2, \ldots, l .
$$

Then, condition (37) of Theorem 7 is guaranteed to hold. 
Note that $\left(-\frac{\partial z_{k}}{\partial p_{k}}\right)^{-\frac{1}{2}}\left(-\frac{\partial z_{T}}{\partial p_{T}}\right)^{\frac{1}{2}} \leq 1$, and, hence, this is a diagonal dominance condition which has been imposed in the literature to ensure the global stability of the tatonnement process (see, e. g., Cornwell (1984)).

Recalling that $\nabla_{p} g(p, q)$ is diagonal and positive definite, and observing that the diagonal elements $-\frac{\partial z_{i}}{\partial p_{i}}$ depend only on $p_{i}$, we see that $\mathrm{VI}^{k}\left(g, S^{l}\right)$ is equivalent to the separable strictly convex mathematical programming problem

$$
\begin{gathered}
\min _{p \in S^{l}} F(p)=\min _{p \in S^{l}}\left\{\int_{0}^{p} g\left(p, p^{k-1}\right)^{T} d p\right\} \\
=\min _{p \in S^{l}}\left\{-\sum_{i=1}^{l} \int_{0}^{p_{i}} z_{i}\left(p_{1}^{k-1}, \ldots, p_{i-1}^{k-1}, p_{i}, q_{i+1}^{k-1}, \ldots, q_{l}^{k-1} d p_{i}\right\}\right.
\end{gathered}
$$

which can be solved, in general, by any efficient mathematical programming algorithm. 
Now WREA is presented for solving $\mathrm{VI}^{k}\left(g, S^{l}\right)$ where $g(\cdot)$ is specified by above, which exploits the special network structure of the problem. For a graphic depiction, see Figure 3.

\section{WREA}

\section{Step 0: Initialization}

Start with the feasible point $p^{k-1} \in S^{l}$ which is obtained by solving $\mathrm{VI}^{k-1}\left(g, S^{l}\right)$ and let $n=k-1$.

\section{Step 1: Selection}

Select $m$ and $s$ such that

$$
g_{m}\left(p^{n}, p^{k-1}\right)=\max _{\left\{i, p_{i}^{n}>0\right\}}\left\{g_{i}\left(p^{n}, p^{k-1}\right)\right\}
$$

or

$$
\begin{gathered}
-z\left(p_{1}^{k-1}, \ldots, p_{m-1}^{k-1}, p_{m}^{n}, p_{m+1}^{k-1}, \ldots, p_{l}^{k-1}\right) \\
=\max _{\left\{i, p_{i}^{n}>0\right\}}\left\{-z_{i}\left(p_{1}^{k-1}, \ldots, p_{i-1}^{k-1}, p_{i}^{n}, p_{i+1}^{k-1}, \ldots, p_{l}^{k-1}\right)\right\} \\
g_{s}\left(p^{n}, p^{k-1}\right)=\min _{i}\left\{g_{i}\left(p^{n}, p^{k-1}\right)\right\},
\end{gathered}
$$


or

$$
\begin{gathered}
-z_{s}\left(p_{1}^{k-1}, \ldots, p_{s-1}^{k-1}, p_{s}^{n}, p_{s+1}^{k-1}, \ldots, p_{l}^{k-1}\right) \\
=\min _{i}\left\{-z_{i}\left(p_{1}^{k-1}, \ldots, p_{i-1}^{k-1}, p_{i}^{n}, p_{i+1}^{k-1}, \ldots, p_{l}^{k-1}\right)\right\} .
\end{gathered}
$$

If $\left|g_{m}\left(p^{n}, p^{k-1}\right)-g_{s}\left(p^{n}, p^{k-1}\right)\right| \leq \epsilon$, for $\epsilon>0$ a preset convergence tolerance, then stop. The current $p^{n}$ is a solution of $\mathrm{VI}^{k}\left(g, S^{l}\right)$. Otherwise, go to Step 2 .

\section{Step 2: Equilibration}

Equilibrate $g_{m}$ and $g_{s}$ by solving the following one-dimensional mathematical programming problem for $\delta$ :

$$
\begin{gathered}
\min z_{m}\left(p_{1}^{k-1}, \ldots, p_{m-1}^{k-1}, p_{m}^{n}-\delta, p_{m+1}^{k-1}, \ldots, p_{l}^{k-1}\right) \\
-z_{s}\left(p_{1}^{k-1}, \ldots, p_{s-1}^{k-1}, p_{s}^{n}+\delta, p_{s+1}^{k-1}, \ldots, p_{l}^{k-1}\right),
\end{gathered}
$$

subject to $0 \leq \delta \leq p_{m}^{n}$. 


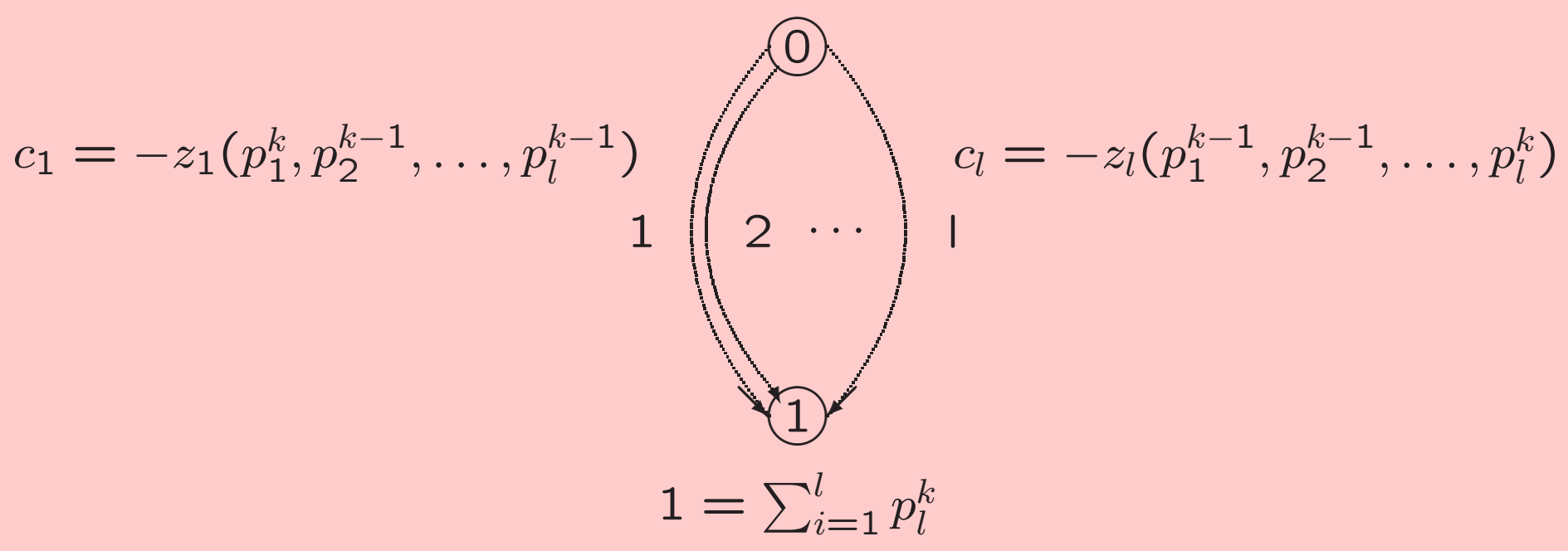

Network equilibrium representation of subproblem induced by the relaxation method 
Suppose that $\delta^{n}$ is the solution of the above minimization problem. Let

$$
\begin{gathered}
p_{i}^{n+1}=p_{i}^{n}, \quad \forall i \neq m, s, \\
p_{m}^{n+1}=p_{m}^{n}-\delta^{n} \\
p_{s}^{n+1}=p_{s}+\delta^{n}
\end{gathered}
$$

and go back to Step 1 with $n=n+1$.

The sequence $\left\{p^{n}\right\}$ obtained in this manner converges to the solution of $\operatorname{VI}^{k}\left(g, S^{l}\right)$, which can be seen by the fact that

$$
F\left(p^{n+1}\right)<F\left(p^{n}\right)
$$

where $F(\cdot)$ is the objective function above.

Convergence condition of the projection method and convergence condition of the relaxation method have the following interpretation: If the price of a commodity is a decreasing function of the demand for this commodity and is affected principally by the demand for the commodity, then these conditions can be expected to hold. 


\section{A Numerical Example}

Here a numerical example is presented which can be solved either by the projection method and the relaxation method. The aggregate excess demand functions in this economy are assumed derived from Cobb-Douglas utility functions and are of the form:

$$
z_{j}(p)=\sum_{i=1}^{m} \frac{p^{T} W^{i}}{\sum_{j=1}^{l} a_{j}^{i}}\left(\frac{a_{j}^{i}}{p_{j}}\right)-\sum_{i=1}^{m} w_{j}^{i}, \quad j=1, \ldots, l,
$$

where $W^{i}$ is the vector with components $\left\{w_{1}^{i}, \ldots, w_{l}^{i}\right\}$. The example is taken from Eaves (1983) and the data are given in Table 1 for ready reproducibility and convenience. The values of $a_{j}^{i}$ and $w_{j}^{i}$ can be found in the cell blocks. In this economy there are eight commodities and five consumers. 


\section{Parameters for a Walrasian price equilibrium example}

\begin{tabular}{|c|c|c|c|c|c|}
\hline$a_{j}^{i}, w_{j}^{i}$ & $i=1$ & $i=2$ & $i=3$ & $i=4$ & $i=5$ \\
\hline$j=1$ & $0.3,3.0$ & $0.0,0.0$ & $0.0,0.0$ & $0.0,0.0$ & $0.0,4.0$ \\
\hline$j=2$ & $0.0,0.0$ & $0.0,15$. & $1.0,0.0$ & $0.0,0.0$ & $0.0,0.0$ \\
\hline$j=3$ & $.13,0.0$ & $0.0,0.0$ & $0.0,0.0$ & $0.0,5.0$ & $0.0,0.0$ \\
\hline$j=4$ & $0.0,3.0$ & $0.0,0.0$ & $0.0,0.0$ & $.73,4.0$ & $.47,13$. \\
\hline$j=5$ & $0.0,3.0$ & $1.0,2.0$ & $0.0,3.0$ & $0.0,0.0$ & $0.0,0.0$ \\
\hline$j=6$ & $0.0,5.0$ & $1.0,0.0$ & $0.0,0.0$ & $0.0,0.0$ & $.11,0.0$ \\
\hline$j=7$ & $.38,2.0$ & $1.0,0.0$ & $0.0,0.0$ & $0.0,4.0$ & $.05,6.0$ \\
\hline$j=8$ & $.19,0.0$ & $1.0,0.0$ & $0.0,0.0$ & $.27,4.0$ & $.37,6.0$ \\
\hline
\end{tabular}


Computation of economic equilibria, thus far, has been typically based either on classical algorithms for solving nonlinear systems of equations (see, e.g., Ginsburgh and Waelbrock (1981)), or on simplicial approximation methods pioneered by Scarf (1973) (see also the contributions of Todd (1976, 1979), Shoven (1983), Whalley (1977), Van der Laan and Talman (1987)). The former techniques are applicable only when the equilibrium lies in the interior of the feasible set, while the latter techniques are general-purpose algorithms and are capable of handling inequality constraints.

However, in their present state of development, they are unable to handle large-scale problems (cf. Mathiesen (1987)). General economic equilibrium problems have been formulated as nonlinear complementarity problems (see Manne (1985)), and a Newton-type method based on this formulation has been used by many researchers for the computation of equilibria (cf. Eaves (1983), Manne and Preckel (1985), Rutherford (1987)).

Although this approach has been proven to be more effective than fixed point methods, its convergence has not been proven theoretically (see, e. g., Mathiesen (1987)). 
Here we provide the references cited in the text as well as additional ones.

\section{References}

Border, K. C., Fixed Point Theorems with Application to Economics and Game Theory, Cambridge University Press, Cambridge, United Kingdom, 1985.

Cornwell, R., Introduction to the Use of General Equilibrium Analysis, North-Holland, Amsterdam, The Netherlands, 1984.

Dafermos, S., "Exchange price equilibria and variational inequalities," Mathematical Programming 46 (1990) 391402.

Debreu, G., The Theory of Value, John Wiley \& Sons, New York, 1959.

Eaves, B. C., "Where solving for stationary points by LCPS is mixing Newton iterates," in Homotopy Methods and Global Convergence, pp. 63-78, B. C. Eaves, F. J. Gould, H. O. Peitgen, and M. J. Todd, editors, Plenum Press, New York, 1983.

Ginsburgh, V., and Waelbrock, J. L., Activity Analysis and General Equilibrium Modelling, Contributions to Economic Analysis 125, North-Holland, Amsterdam, The Netherlands, 1981. 
Manne, A. S., "On the formulation and solution of economic equilibrium models," Mathematical Programming Study 23 (1985) 1-22.

Manne, A. S., and Preckel, P. V., "A three-region intertemporal model of energy, international trade, and capital flows," Mathematical Programming Study 23 (1985) 56-74.

Mas-Colell, A., The Theory of General Economic Equilibrium: A Differentiable Approach, Econometric Society Monographs 9, Cambridge University Press, Cambridge, United Kingdom, 1985.

Mathiesen, L., "An algorithm based on a sequence of linear complementarity problems applied to a Walrasian equilibrium model: an example," Mathematical Programming 37 (1987) 1-18.

Rutherford, T., "A modeling system for applied general equilibrium analysis," Cowles Foundation Discussion Paper No. 836, Yale University, New Haven, Connecticut, 1987.

Scarf, H. (with T. Hansen), Computation of Economic Equilibria, Yale University Press, New Haven, Connecticut, 1973. 
Shoven, J. B., "The application of fixed point methods to economics," in Homotopy Methods and Global Convergence, pp. 249-262, B. C. Eaves, F. J. Gould, H. O. Peitgen, and M. J. Todd, editors, Plenum Press, New York, 1983.

Todd, M. J., The Computation of Fixed Points and Applications, Lecture Notes in Economics and Mathematical Systems 124, Springer-Verlag, Berlin, Germany, 1976.

Todd, M. J., "A note on computing equilibria in economics with activity models of production," Journal of Mathematical Economics 6 (1979) 135-144.

Van der Laan, G., and Talman, A. J. J., "Simplicial approximation of solutions to the nonlinear complementarity problem with lower and upper bounds," Mathematical Programming 38 (1987) 1-15.

Wald, A., "On some systems of equations in mathematical economics," Econometrica 19 (1951) 368-403.

Walras, L., Elements d'Economique Politique Pure, Corbaz, Lausanne, Switzerland, 1874.

Whalley, J., "Fiscal harmonization in the EEC: some preliminary findings of fixed point calculations," in Fixed Points: Algorithms and Applications, pp. 435-472, S. Karamardian and C. B. Garcia, editors, Academic Press, New York, 1977. 
Zhao, L., "Variational inequalities in general equilibrium: analysis and computation," Ph. D. Thesis, Division of Applied Mathematics, Brown University, Providence, Rhode Island, 1989, also appears as: LCDS \# 88-24, Lefschetz Center for Dynamical Systems, Brown University, Providence, Rhode Island, 1988.

Zhao, L., and Dafermos, S., "General economic equilibrium and variational inequalities," Operations Research Letters 10 (1991) 369-376.

Zhao, L., and Nagurney, A., "A network formalism for pure exchange economic equilibria," in Network Optimization Problems: Algorithms, Complexity, and Applications, pp. 363-386, D. Z. Du and P. M. PardaIos, editors, World Scientific Press, Singapore, 1993. 